\title{
Impact of cosmic variance on the local measurement of $H_{0}$
}

\author{
Giovanni Marozzi*1, Ido Ben-Dayan ${ }^{2}$, Ruth Durrer ${ }^{1}$, Dominik J. Schwarz ${ }^{3}$ \\ ${ }^{1}$ Université de Genève, Département de Physique Théorique and CAP, 24 quai Ernest-Ansermet, \\ CH-1211 Genève 4, Switzerland \\ ${ }^{2}$ Deutsches Elektronen-Synchrotron DESY, Theory Group, D-22603 Hamburg, Germany \\ ${ }^{3}$ Fakultät für Physik, Universität Bielefeld, Postfach 100131, 33501 Bielefeld, Germany \\ E-mail: giovanni.marozzieunige.ch
}

\begin{abstract}
Local measurements of the Hubble expansion rate are affected by structures like galaxy clusters or voids. Here we present a fully relativistic treatment of this effect, studying how clustering modifies the dispersion of the mean distance (modulus)-redshift relation in a standard $\Lambda \mathrm{CDM}$ universe. Our findings is that cosmic variance (i.e. the effects of the local structure) is, for supernova observations at small redshifts $(0.01<z<0.1)$, of the same order of magnitude as the current observational errors. The cosmic variance has to be taken into account in local measurements of the Hubble expansion rate and it reduces the tension with the CMB measurement.
\end{abstract}

Frontiers of Fundamental Physics 14

15-18 July 2014

Aix Marseille University (AMU) Saint-Charles Campus, Marseille, France

${ }^{*}$ Speaker. 


\section{Introduction}

The Hubble constant, $H_{0}$, determines the present expansion rate of the Universe. For most cosmological phenomena a precise knowledge of $H_{0}$ is of utmost importance. In a perfectly homogeneous and isotropic world $H_{0}$ is defined globally. But the Universe contains structures like galaxy clusters and voids. Thus the local expansion rate, measured by means of cepheids and supernovae at small redshifts, does not necessarily agree with the expansion rate of an isotropic and homogeneous model that is used to describe the Universe at the largest scales.

Recent local measurements of the Hubble rate $[1,2]$ are claimed to be accurate at the few percent level, e.g. [1] finds $H_{0}=(73.8 \pm 2.4) \mathrm{km} \mathrm{s}^{-1} \mathrm{Mpc}^{-1}$. In the near future, observational techniques will improve further, such that the local value of $H_{0}$ will be determined at $1 \%$ accuracy [3], competitive with the current precision of indirect measurements of the global $H_{0}$ via the cosmic microwave backgound anisotropies [4].

The observed distance modulus $\mu$ is related to the bolometric flux $\Phi$ and the luminosity distance $d_{\mathrm{L}}$ by $\left(\log \equiv \log _{10}\right)$

$$
\mu=-2.5 \log \left[\Phi / \Phi_{10 \mathrm{pc}}\right]=5 \log \left[d_{\mathrm{L}} /(10 \mathrm{pc})\right] .
$$

The relation between the intrinsic luminosity, $L$, the bolometric flux, $\Phi$, and the luminosity distance $d_{L}$ of a source is $\Phi=L / 4 \pi d_{L}^{2}$. In a flat $\Lambda \mathrm{CDM}$ universe with present matter density parameter $\Omega_{m}$ the luminosity distance as a function of redshift $z$ is given by

$$
d_{L}(z)=\frac{1+z}{H_{0} / c} \int_{0}^{z} \frac{d z^{\prime}}{\sqrt{\Omega_{m}\left(1+z^{\prime}\right)^{3}+1-\Omega_{m}}}
$$

As long as we consider only small redshifts, $z \leq 0.1$, the dependence on cosmology is weak, $d_{L}(z) \simeq$ $c\left[z+\left(1-3 \Omega_{m} / 4\right) z^{2}\right] / H_{0}$ and the result varies by about $0.2 \%$ when $\Omega_{m}$ varies within the $2 \sigma$ error bars determined by Planck [4]. However, neglecting the model dependent quadratic term induces an error of nearly $8 \%$ for $z \simeq 0.1$.

The observed Universe is inhomogeneous and anisotropic on small scales and the local Hubble rate is expected to differ from its global value for two reasons. First, any supernova (SN) sample is finite (sample variance) and, second, we observe only one realization of a random configuration of the local structure (cosmic variance). Thus, even for arbitrarily precise measurements of fluxes and redshifts, the local $H_{0}$ differs from the global $H_{0}$. Sample variance was fully taken into account in the literature, while cosmic variance was addressed in a fully relativistic way only recently in [5].

In the context of Newtonian cosmology, cosmic variance of the local $H_{0}$ has been estimated in $[6,7,8,9]$. First attempts to estimate cosmic variance of the local Hubble rate in a relativistic approach can be found in $[10,11]$ (see also [12]), based on the ensemble variance of the expansion rate averaged over a spatial volume. It has been shown that this approach agrees very well with the Newtonian one [10] and it predicts a cosmic variance which depends on the sampling volume on the sub-per cent to per cent level. However, this approach still neglects the fact that observers probe the past light-cone and not a spatial volume. Also, the measured quantity is not an expansion rate, but a set of the bolometric fluxes and redshifts.

Here, we present the results obtained [5], where the first fully relativistic estimation of the effects of clustering on the local measurement of the Hubble parameter, without making any special hypothesis about how the fluctuations can be modeled around us, was given. In [5], considering only the measured quantities and the cosmological standard model with stochastic inhomogeneities, 
was studied the effect of cosmic structures on the local determination of $H_{0}$, i.e., the light propagation effects were taken fully into account. Other relativistic approaches were proposed in [13, 14]. In [13] a "Swiss cheese" model was used in modeling the local Universe, in [14] a "Hubble bubble" model was used and the perturbation of the expansion rate, which is not directly measurable, was considered.

Following [5], we shall find that the contribution from clustering to the error budget of the mean value of the Hubble parameter is typically 2 to $3 \%$, hence as large as observational errors quoted in the literature [1]. Finally, we shall find that even for an infinite number of SNIa within $0.01<z<0.1$ with identical redshift distribution compared to a finite sample considered, clustering induces a minimal error of about $2 \%$ for a local determination of $H_{0}$.

The paper is organized as follows. In Sec. 2 we consider the effect of local structure on the error budget associated to the measurement of the local value of the Hubble parameter for a given sample of SNe. Then, in Sec 3 we generalize this result to the case in which we have an infinite number of SNe in our sample. Finally, we conclude in Sec. 4.

\section{Cosmic Variance from local structure: finite SNe sample}

Following $[15,16]$ one can use cosmological perturbation theory up to second order with an almost scale-invariant initial power spectrum to determine the mean perturbation of the bolometric flux from a standard candle and its variance.

Let us first consider the fluctuation of the mean on a sphere at fixed observed redshift $z$. We denote the light-cone average [17] over a surface at fixed redshift by $\langle\cdots\rangle$, and a statistical average by $\cdots$. Using the results of $[18,19,20]$ (see also [21]) the fluctuation of the flux $\Phi \propto d_{L}^{-2}$, away from its background value in the Friedmann-Lemaître Universe (denoted by $\left(d_{L}^{\mathrm{FL}}\right)^{-2}$ ), is given by

$$
d_{L}^{-2}=\left(d_{L}^{\mathrm{FL}}\right)^{-2}\left[1+\Phi_{1} / \Phi_{0}+\Phi_{2} / \Phi_{0}\right],
$$

where we expand $\Phi=\Phi_{0}+\Phi_{1}+\Phi_{2}$ up to second order in perturbation theory. The ensemble average of $\left\langle\Phi_{1} / \Phi_{0}\right\rangle$ vanishes at first order, but not at second order and must be added to another second order contribution from $\Phi_{2} / \Phi_{0}$; we obtain (see, e.g. [16])

$$
\overline{\left\langle d_{L}^{-2}\right\rangle}(z)=\left(d_{L}^{\mathrm{FL}}\right)^{-2}\left[1+f_{\Phi}(z)\right]
$$

where for $z \ll 1$

$$
f_{\Phi}(z) \simeq-\left(\frac{1}{\mathscr{H}(z) \Delta \eta}\right)^{2} \overline{\left\langle\left(\vec{v}_{s} \cdot \vec{n}\right)^{2}\right\rangle} .
$$

Here $\vec{n}$ denotes the direction to a given $\mathrm{SN}$ and $\vec{v}_{s}$ its peculiar velocity, $\eta$ is conformal time, $\Delta \eta=\eta_{0}-\eta(z)$ is the difference between the present time and the time at redshift $z$, and $\mathscr{H}$ is the conformal Hubble parameter. We have removed the observer velocity since observations are usually quoted in the CMB frame, corresponding to $\vec{v}_{0}=0$. A non-vanishing observer velocity would nearly double the effect in Eq. (2.3).

The variance associated to the mean value of the flux in Eq.(2.2) is then given by [22]

$$
\sigma_{\Phi}^{2}=\overline{\left\langle\left(\Phi_{1} / \Phi_{0}\right)^{2}\right\rangle}
$$

at low redshift we have the following dominant peculiar velocity contribution

$$
\sigma_{\Phi}^{2} \simeq 4\left(\frac{1}{\mathscr{H}(z) \Delta \eta}\right)^{2} \overline{\left\langle\left(\vec{v}_{s} \cdot \vec{n}\right)^{2}\right\rangle} .
$$


Finally, in Fourier space and using the dimensionless power spectrum of the Bardeen potential today, $\mathscr{P}_{\psi}(k)=\left(k^{3} / 2 \pi^{2}\right)\left|\Psi_{k}\left(\eta_{0}\right)\right|^{2}$, we have

$$
\sigma_{\Phi}^{2} \simeq 4\left(\frac{1}{\mathscr{H}(z) \Delta \eta}\right)^{2} \frac{\tau^{2}(z)}{3} \int_{H_{0}}^{k_{\mathrm{UV}}} \frac{d k}{k} k^{2} \mathscr{P}_{\psi}(k),
$$

where

$$
\tau(z)=\int_{\eta_{\text {in }}}^{\eta_{s}} d \eta \frac{a(\eta)}{a\left(\eta_{s}\right)} \frac{g(\eta)}{g\left(\eta_{0}\right)} .
$$

$g(\eta)$ is the growth factor and the source and the observer times are indicated with the suffix $s$ and 0 .

The approximate equalities in Eqs. (2.3) and (2.5) are valid for $z \ll 1$, where the first order squared contribution of the peculiar velocity terms dominates over the other second order contributions. For $z \sim 0.3$ and larger, additional contributions notably due to lensing become relevant, see $[15,16]$.

For measurements of the Hubble parameter, low redshift $\mathrm{SNe}$ are used in order to minimize the dependence of the result on cosmological parameters. As a consequence, Eq. (2.5) is a good approximations for the case here under investigation.

Hereafter we use the cosmological parameters from Planck [4], the linear transfer function given in [23] taking baryons into account, and $k_{\mathrm{UV}}=0.1 \mathrm{~h} \mathrm{Mpc}^{-1}$, see [16] for details. Increasing the cut-off does not change the results here described due to two effects: the kernel $k^{2} \mathscr{P}_{\psi}(k)$ of the peculiar velocity contribution decreases at large $k$ and small scale fluctuations are incoherent (see below) and their contribution to the variance decays like $1 / N$, where $N$ is the number of supernovae.

The brightness of supernovae is typically expressed in terms of the distance modulus $\mu$. On the other hand, the induced theoretical dispersion on the bare value of $H_{0}$, which is entirely due to squared first order perturbations, is independent of the observable considered to infer $H_{0}$. To determine the dispersion of $H_{0}$ from a sample of SNe we consider that at small redshift $H_{0}^{2} \simeq$ $c^{2} z^{2} / d_{L}^{2} . H_{0}$ inferred from the observation of a single $\mathrm{SN}$ at redshift $z \ll 1$, is then expected to deviate from the true $H_{0}$ by approximately [22]

$$
\left(\Delta H_{0}\right)^{2}=\frac{H_{0}^{2}}{4} \overline{\left\langle\left(\Phi_{1} / \Phi_{0}\right)^{2}\right\rangle} .
$$

In practice, observers do not have at their disposal many $\mathrm{SNe}$ at the same redshift, so the average over a sphere cannot be performed. Hence, one has to go beyond this simplifying assumption.

Following [5], let us estimate the (ensemble) variance of the locally measured Hubble parameter $H_{0}$ from the covariance matrix of the fluxes, given an arbitrarily distributed sample of $N$ observed $\mathrm{SNe}$ at positions $\left(z_{i}, \vec{n}_{i}\right)$, which reads

$$
\begin{aligned}
\left(\frac{\Delta H_{0}}{H_{0}}\right)^{2} & =\frac{1}{4 N^{2}} \sum_{i j} \frac{\overline{\Phi_{1}\left(z_{i}, \vec{n}_{i}\right)}}{\Phi_{0}\left(z_{i}\right)} \frac{\Phi_{1}\left(z_{j}, \vec{n}_{j}\right)}{\Phi_{0}\left(z_{j}\right)} \\
& =\frac{1}{N^{2}} \sum_{i j} \frac{V_{i j}}{\mathscr{H}\left(z_{i}\right) \Delta \eta_{i} \mathscr{H}\left(z_{j}\right) \Delta \eta_{j}}
\end{aligned}
$$

with

$$
V_{i j}=\tau\left(z_{i}\right) \tau\left(z_{j}\right) \int_{H_{0}}^{k_{\mathrm{Uv}}} \frac{d k}{k} k^{2} \mathscr{P}_{\psi}(k) I\left(k \Delta \eta_{j}, k \Delta \eta_{i},\left(\vec{n}_{i} \cdot \vec{n}_{j}\right)\right)
$$




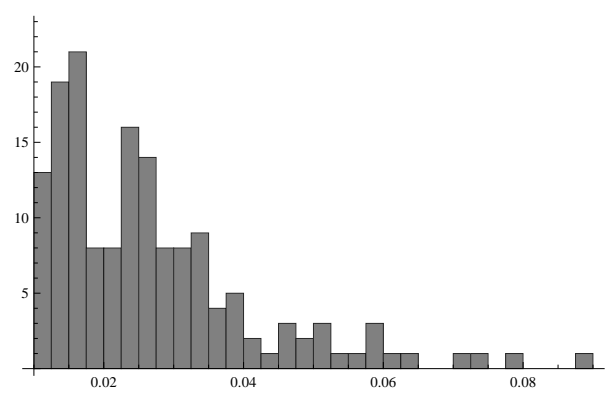

Figure 1: The redshift distribution of the $155 \mathrm{SNe}$ of the CfA3+OLD sample [24, 25] with redshift within 0.01 and 0.1 considered here.

and

$$
\begin{aligned}
I(x, y, v) & =\frac{1}{4 \pi} \int d \Omega_{\hat{k}} e^{i x\left(\hat{k} \cdot \vec{n}_{i}\right)} e^{-i y\left(\hat{k} \cdot \vec{n}_{j}\right)}\left(\hat{k} \cdot \vec{n}_{j}\right)\left(\hat{k} \cdot \vec{n}_{i}\right) \\
& =\frac{x y\left(1-v^{2}\right)}{R^{2}} j_{2}(R)+\frac{v}{3}\left[j_{0}(R)-2 j_{2}(R)\right],
\end{aligned}
$$

where $v=\left(\vec{n}_{i} \cdot \vec{n}_{j}\right)$ and $R=\sqrt{x^{2}+y^{2}-2 v x y}=k d$. Here $d$ is the comoving distance between the $\mathrm{SNe}$ at $\left(z_{i}, \vec{n}_{i}\right)$ and $\left(z_{j}, \vec{n}_{j}\right), j_{\ell}$ denotes the spherical Bessel function of order $\ell$ and $\hat{k}$ is the unit vector in direction $\vec{k}$. To arrive at (2.10), one has to introduce the Fourier representation of $\Phi_{1}\left(z_{i}, \vec{n}_{i}\right)=$ $2 /\left(\mathscr{H}\left(z_{i}\right) \Delta \eta_{i}\right) \vec{v}_{s}(\vec{k}) \cdot \vec{n}_{i}$ and use some well known identities. Note that with $I(x, x, 1)=1 / 3$ and Eq.(2.6), the auto-correlation term reproduces (2.7).

If the fluxes are perfectly coherent for all SNe so that $\overline{\Phi_{1}\left(z_{i}, \vec{n}_{i}\right) \Phi_{1}\left(z_{j}, \vec{n}_{j}\right)}=4 \sigma^{2} \Phi_{0}\left(z_{j}\right) \Phi_{0}\left(z_{i}\right)$, for all correlations, we obtain $\left(\Delta H_{0} / H_{0}\right)^{2}=\sigma^{2}$, while in the incoherent case, $\overline{\Phi_{1}\left(z_{i}, \vec{n}_{i}\right) \Phi_{1}\left(z_{j}, \vec{n}_{j}\right)}=$ $\delta_{i j} 4 \sigma^{2} \Phi_{0}\left(z_{j}\right) \Phi_{0}\left(z_{i}\right)$ we obtain $\left(\Delta H_{0} / H_{0}\right)^{2}=\sigma^{2} / N$. The reality lies somewhere in-between, wavelengths with $k d<1$ being rather coherent while those with $k d>1$ are rather incoherent.

In order to estimate the effect of the cosmic (co)variance for a realistic sample of $\mathrm{SNe}$, we consider the following set up. We calculate $\Delta H_{0} / H_{0}$ from Eqs. (2.8) to (2.10) considering the redshifts of a sample of $155 \mathrm{SNe}$ selected to lie in the range $0.01 \leq z \leq 0.1$ from the CfA3 and OLD samples [24, 25]. The redshift distribution of the sample is shown in Fig 1.

For the redshift distribution of the $155 \mathrm{SNe}$ of this sample, Eq. (2.8) yields a dispersion induced by inhomogeneities between 2.2 and $3.3 \%$ for different angular distributions for the SNe. From this range we infer [5]

$$
\Delta H_{0}=(1.6 \div 2.4) \mathrm{km} \mathrm{s}^{-1} \mathrm{Mpc}^{-1},
$$

with $H_{0}$ as given in [1]. To obtain the result in Eq. (2.11) one has to keep $v$ constant to different values and chose a random distribution of directions over one hemisphere. The different choices give rise to the range quoted above. The smallest error corresponds to a random distribution of directions over one hemisphere, while the largest one corresponds to the case where all $\mathrm{SNe}$ are inside a narrow cone $(v \simeq 1)$.

Considering the quoted observational error of $2.4 \mathrm{~km} / \mathrm{s} / \mathrm{Mpc}$ [1] and the additional variance (2.11), we obtain

$$
H_{0}=[73.8 \pm 2.4 \pm(1.6 \div 2.4)] \mathrm{km} \mathrm{s}^{-1} \mathrm{Mpc}^{-1} .
$$

The tension with the Planck measurement [4], for which a value $\left(H_{0}\right)_{\mathrm{CMB}}=67.3 \pm 1.2 \mathrm{~km} \mathrm{~s}^{-1} \mathrm{Mpc}^{-1}$ is reported, is reduced when taking this additional variance into account. In particular, adding the 
above errors in quadrature one obtains a deviation of 2.2 to $1.9 \sigma$ from $\left(H_{0}\right)_{\mathrm{CMB}}$, while the difference is $2.7 \sigma$ when using the error quoted in [1]. This analysis is insensitive to smaller scales fluctuations due to the incoherence of such contributions.

\section{Cosmic Variance from local structure: infinite SNe sample}

Following [5], let us now look at the ultimate error for an arbitrarily large sample when the $\mathrm{SNe}$ are distributed isotropically over directions. In this case we can integrate $I(x, y, v)$ over all directions. With

$$
\frac{1}{2} \int_{-1}^{1} d v I(x, y, v)=j_{1}(x) j_{1}(y)
$$

we then obtain [5]

$$
\left(\frac{\Delta H_{0}}{H_{0}}\right)^{2}=\int \frac{d k}{k} k^{2} \mathscr{P}_{\psi}(k)\left(\int d z \tau(z) s(z) \frac{j_{1}(k \Delta \eta(z))}{\mathscr{H}(z) \Delta \eta(z)}\right)^{2}
$$

with $\int d z s(z)=1$. Approximating the redshift distribution of the sample considered using an interpolating function of the histogram in Fig 1, and integrating from $z=0.01$ to 0.1, we obtain a dispersion of about $1.8 \%$ which corresponds to an error of

$$
\Delta H_{0}=1.3 \mathrm{~km} \mathrm{~s}^{-1} \mathrm{Mpc}^{-1} .
$$

This is the minimal dispersion of a SN sample with a redshift space distribution given by the one in Fig 1. It is not much smaller than the value obtained for the real sample. Interestingly, this result is close to the ones obtained in $[9,10,14]$, some of them with a very different analysis.

The errors from the nearby SNe with small $\Delta \eta(z)$ give the largest contribution. Therefore, the dispersion can be reduced by considering higher redshift $\mathrm{SNe}$ for which, however, the model dependence becomes more relevant. If we consider higher redshifts (close to or larger than 0.3), we have to take into account also the other contributions to the perturbation of the luminosity distance, see $[18,19,20,21]$ for the full expression. As it is well known (see, for example, $[15,16])$, at redshift $z>0.3$, the lensing term begins to dominate.

\section{Conclusion}

To conclude, following [5] we have described the impact of stochastic inhomogeneities on the error budget associated to the measurement of the local value of the Hubble parameter for a given sample of standard candles. Eqs. (2.8) to (2.10) and (3.1) are the main result here described, namely a general formula for the cosmic variance contribution to $\Delta H_{0}$ from a sample of SNe with $z \lesssim 0.2$, where the Doppler term dominates, and its limit for an arbitrarily large number of $\mathrm{SNe}$ isotropically distributed over directions. This general formula can be easily implemented and does not require an N-body simulation for each set of cosmological parameters. The required input are solely the linear power spectrum and the distribution of the observed SNe in position and redshift space. In particular, for samples presently under consideration, this error is not negligible but of the same order as the experimental error, i.e. between 2.2 and 3.3\%. This cosmic variance is a fundamental barrier on the precision of a local measurement of $H_{0}$. It has to be added to the observational uncertainties and it reduces the tension with the CMB measurement of $H_{0}$ [4].

Finally, even when the number of SNe is arbitrarily large, an irreducible error remains due to cosmic variance of the local Universe. This error is about $1.8 \%$ for SNe with redshift $0.01<z<0.1$ 
and a distribution given by the one in Fig.2 (see [5]). This can only be reduced by considering $\mathrm{SNe}$ with higher redshifts, but if too high redshifts are included the result becomes strongly dependent on other cosmological parameters like $\Omega_{m}$ and curvature.

\section{References}

[1] A. G. Riess, L. Macri, S. Casertano, H. Lampeitl, H. C. Ferguson, A. V. Filippenko, S. W. Jha and W. Li et al., Astrophys. J. 730, 119 (2011) [Erratum-ibid. 732, 129 (2011)].

[2] W. L. Freedman, B. F. Madore, V. Scowcroft, C. Burns, A. Monson, S. E. Persson, M. Seibert and J. Rigby, Astrophys. J. 758, 24 (2012).

[3] S. H. Suyu, T. Treu, R. D. Blandford, W. L. Freedman, S. Hilbert, C. Blake, J. Braatz and F. Courbin et al., arXiv:1202.4459 [astro-ph.CO].

[4] P. A. R. Ade et al. [Planck Collaboration], Astron. Astrophys. 571, A16 (2014).

[5] I. Ben-Dayan, R. Durrer, G. Marozzi and D. J. Schwarz, Phys. Rev. Lett. 112, 221301 (2014).

[6] X. -D. Shi and M. S. Turner, Astrophys. J. 493, 519 (1998).

[7] Y. Wang, D. N. Spergel and E. L. Turner, Astrophys. J. 498, 1 (1998).

[8] T. Buchert, M. Kerscher and C. Sicka, Phys. Rev. D 62, 043525 (2000).

[9] R. Wojtak, A. Knebe, W. A. Watson, I. T. Iliev, S. Hess, D. Rapetti, G. Yepes and S. Gottloeber, Mon. Not. Roy. Astron. Soc. 438, 1805 (2014).

[10] N. Li and D. J. Schwarz, Phys. Rev. D 78, 083531 (2008).

[11] A. Wiegand and D. J. Schwarz, Astron. Astrophys. 538, A147 (2012).

[12] C. Clarkson, K. Ananda and J. Larena, Phys. Rev. D 80, 083525 (2009); O. Umeh, J. Larena and C. Clarkson, JCAP 1103, 029 (2011).

[13] P. Fleury, H. Dupuy and J. P. Uzan, Phys. Rev. D 87, 123526 (2013); Phys. Rev. Lett. 111, 091302 (2013).

[14] V. Marra, L. Amendola, I. Sawicki and W. Valkenburg, Phys. Rev. Lett. 110, 241305 (2013).

[15] I. Ben-Dayan, M. Gasperini, G. Marozzi, F. Nugier and G. Veneziano, Phys. Rev. Lett. 110, 021301 (2013).

[16] I. Ben-Dayan, M. Gasperini, G. Marozzi, F. Nugier and G. Veneziano, JCAP 06, 002 (2013).

[17] M. Gasperini, G. Marozzi, F. Nugier and G. Veneziano, JCAP 07, 008 (2011).

[18] I. Ben-Dayan, G. Marozzi, F. Nugier and G. Veneziano, JCAP 11, 045 (2012).

[19] G. Fanizza, M. Gasperini, G. Marozzi and G. Veneziano, JCAP 11, 019 (2013).

[20] G. Marozzi, Class. Quant. Grav. 32, 045004 (2015).

[21] C. Bonvin, R. Durrer and M. A. Gasparini, Phys. Rev. D 73, 023523 (2006) [Erratum-ibid. D 85, 029901 (2012)].

[22] I. Ben-Dayan, M. Gasperini, G. Marozzi, F. Nugier and G. Veneziano, JCAP 04, 036 (2012).

[23] D. J. Eisenstein, W. Hu, Astrophys. J. 496, 605 (1998).

[24] M. Hicken, P. Challis, S. Jha, R. P. Kirsher, T. Matheson, M. Modjaz, A. Rest and W. M. Wood-Vasey, Astrophys. J. 700, 331 (2009).

[25] S. Jha, A. G. Riess and R. P. Kirshner, Astrophys. J. 659, 122 (2007). 